\title{
Experimental Investigation of Innovative Thermal Mechanical Refrigeration System
}

Abstract
The current electrical refrigeration and air condition systems are considered as
one of the major sources for ozone depletion and global warming problems.
Furthermore, the consume a large percent of the worldwide gross production
of electricity (around 17\%). Therefore, developing new refrigeration systems
that able to work using renewable sources (solar, geothermal, etc) and or
waste heat sources is necessary to address these problems. In this poster, the
experimental investigation of an innovative thermal-mechanical refrigeration
(TMR) system is presented. The TMR system replaces the electric compressor
of the conventional refrigeration systems with an innovative expander-
compressor unit (two connected double-acting cylinders). The proposed ECU
can be driven by ultra-low heat temperature sources, has simple
configuration, and high flexibility for the operating conditions. A hybrid
electric-compressor and ECU refrigeration setup was developed to investigate
the performance of the ECU and compare it to that of an electric compressor.
The experiment was conducted using R134a as a working fluid at different
masses. The results shows that a maximum COP of 0.57 is obtained at
refrigerant mass of $30 \mathrm{~g}$ (in electric mode) and a maximum COP of 0.41 and is
obtained at refrigerant mass of $60 \mathrm{~g}$ (in ECU mode).

\section{Introduction}

- Traditional refrigeration systems use $\mathrm{HFO} / \mathrm{HFC}$ refrigerant blends which have negative impacts on environment such as ozone depletion and global warming [1-4,12-13]. Furthermore, refrige

wide electricity [5]

Therefore, developing new refrigeration systems that work by sustainable energy sources and use eco-friendly refrigerant are urgent need to address these issues [14-16]

Recently, refrigeration systems that use thermal energy as a power source (such as absorption and ejector-based systems) have gained much interest by researchers in the field. However, most of the proposed thermal refrigerating systems have complex configurations, low COP at low temperature source, and comparatively high capital costs [6-8].

Recently, Sleiti et al. [9] have proposed a new thermal mechanical refrigeration (TMR) system that efficiently works with ultra-low temperature source (as low as $70^{\circ} \mathrm{C}$ ) with efficiency higher than $9 \%$

Thus, the proposed TMR system can efficiently works by versatile sources of energy such as solar, geothermal, and waste heat sources. Also, the proposed TMR system use eco-friendly refrigerant with zero ozone depletion potential [10].

- Moreover, the new TMR system has simple configuration and competitive capital cost compared to the conventional refrigeration systems [11].

testing for a lesting for a hybrid electric-TMR setup which is built by the authors in Qatar University.

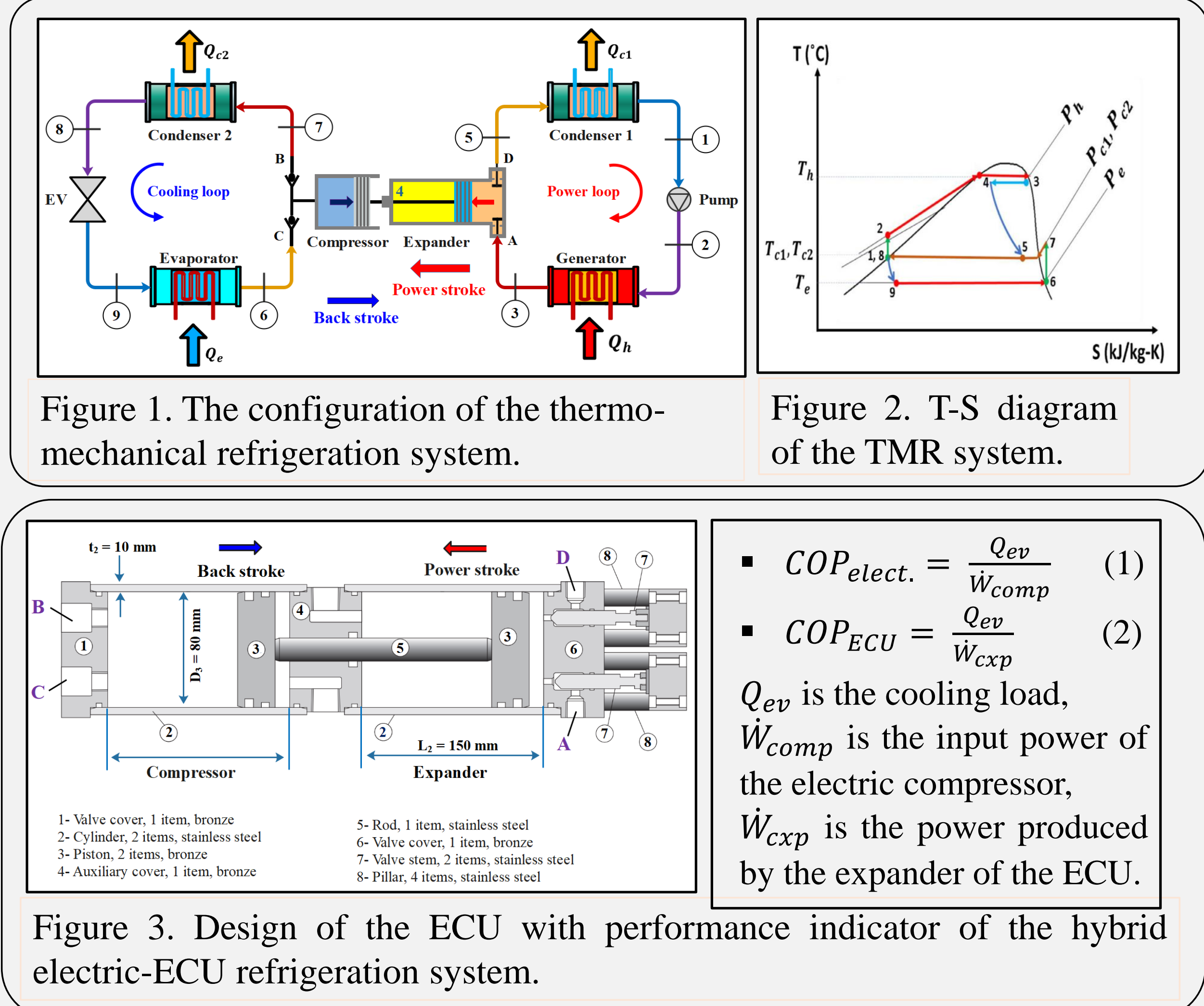

\section{Description}

The proposed TMR system composed of integrated power and cooling loops as shown in Figure 1.

Instead of electric compressor, the TMR system use an the refrigerant of the cooling loop to the condenser pressse (states 6-7 in Figure 2).

- The ECU consists of double acting pistons with interni piston as presented in Figure 3 .

- To test the performance of the ECU and compared it to that of an electric compressor, a hybrid electric-pneumaticmechanical (HEPM) setup is built as shown in Figure 4 and Figure 5.

The HEPM setup exactly simulate the working mechanism of the theoretical TMR system shown in Figure 1

The power loop is replaced with high-pressure the HEPM setup to provide the required pressey to the expander of the ECU.

\section{Experiment setup}

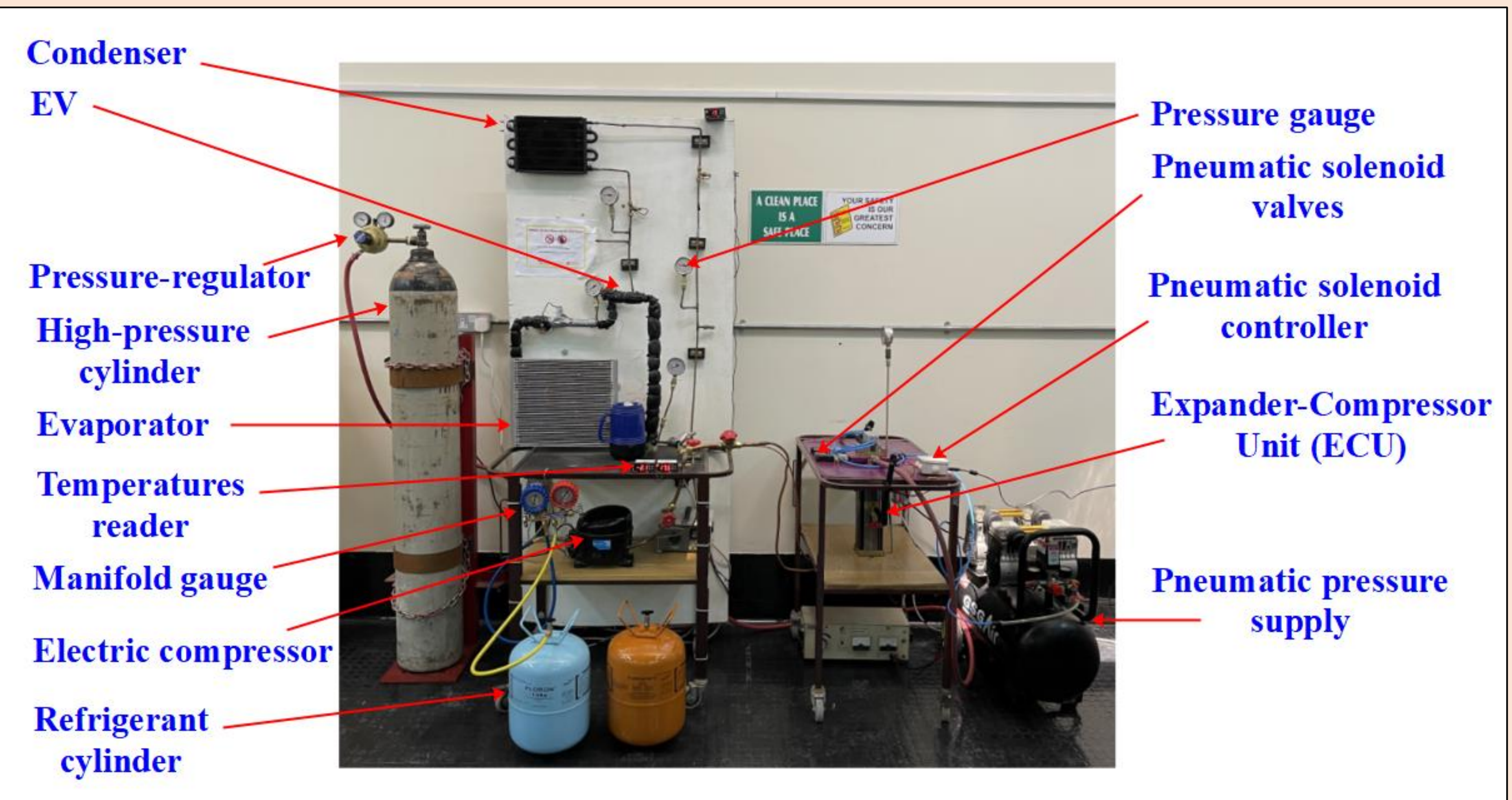

Figure 4. View of the actual setup in the research lab.

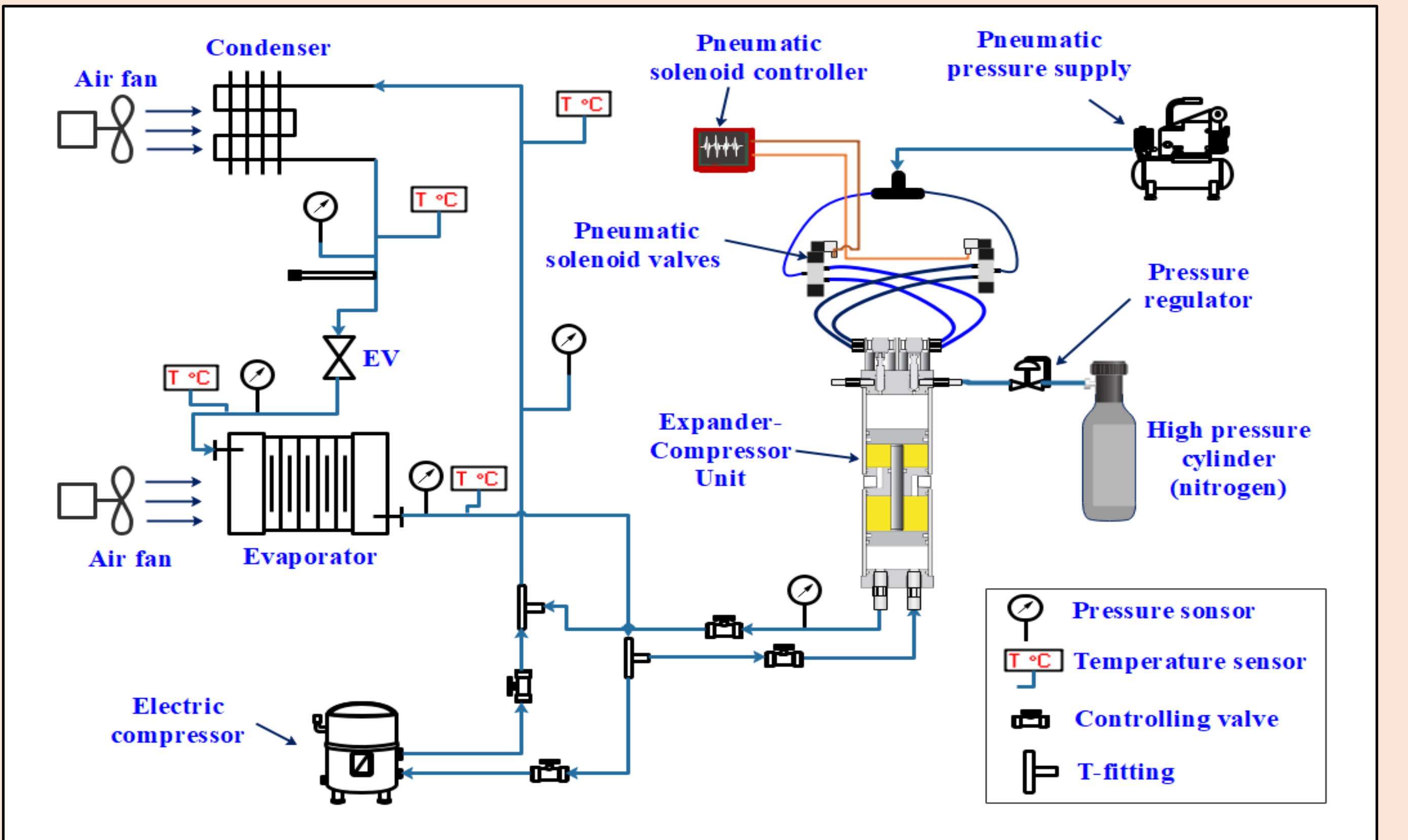

Figure 5. Schematic diagram of the hybrid electric and ECU experimental setup.

\section{Results and discussion}

Figure 6 shows the testing procedures which are applied to test and me The experiment was carried out using R134a as a refrigerant at different The experiment was carried out using R134a as a refrigerant at differen The measured experimental results were validated by comparing them
results as shown in Figure 7 (a) and (b) for electric and ECU, respectively. In electric-compressor mode, the error of the measured values compared to the theoretical values ranges from $0.73 \%$ to $1.85 \%$ while in ECU mode range from $4.50 \%$ to $4.80 \%$ which is acceptable since the

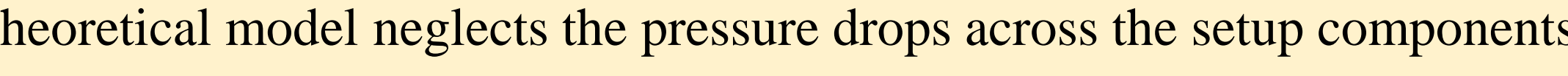

Figure 9 and Figure 10 show the calculated $C O P$ and $\mathrm{Q}_{\mathrm{ev}}$ of the electric-compressor mode and ECU mode, respectively, under the real measured temperatures and pressures at the terminals of the condenser and evaporator of the cooling loop. Figure 8 compares the electric-compressor and ECU modes under the conditions shown figures 9 and $10(\mathrm{~b}$ and $\mathrm{c}$ ).

In electric-compressor mode, a maximum COP of 0.57 and $\mathrm{Q}_{\mathrm{ev}}$ of $0.68 \mathrm{~kW}$ are obtained at refrigerant mass of $30 \mathrm{~g}$. While in ECU mode, a maximum COP of 0.41 and $\mathrm{Q}_{\mathrm{ev}}$ of $0.26 \mathrm{~kW}$ are obtained at refrigerant mass of $60 \mathrm{~g}$.

2.61 times, respectively. However, the frequency of the ECU is only 18rpm which is ultra-low frequency compared to that of the electric compressor (>2000 rpm)

\section{Results of electric operational mode}

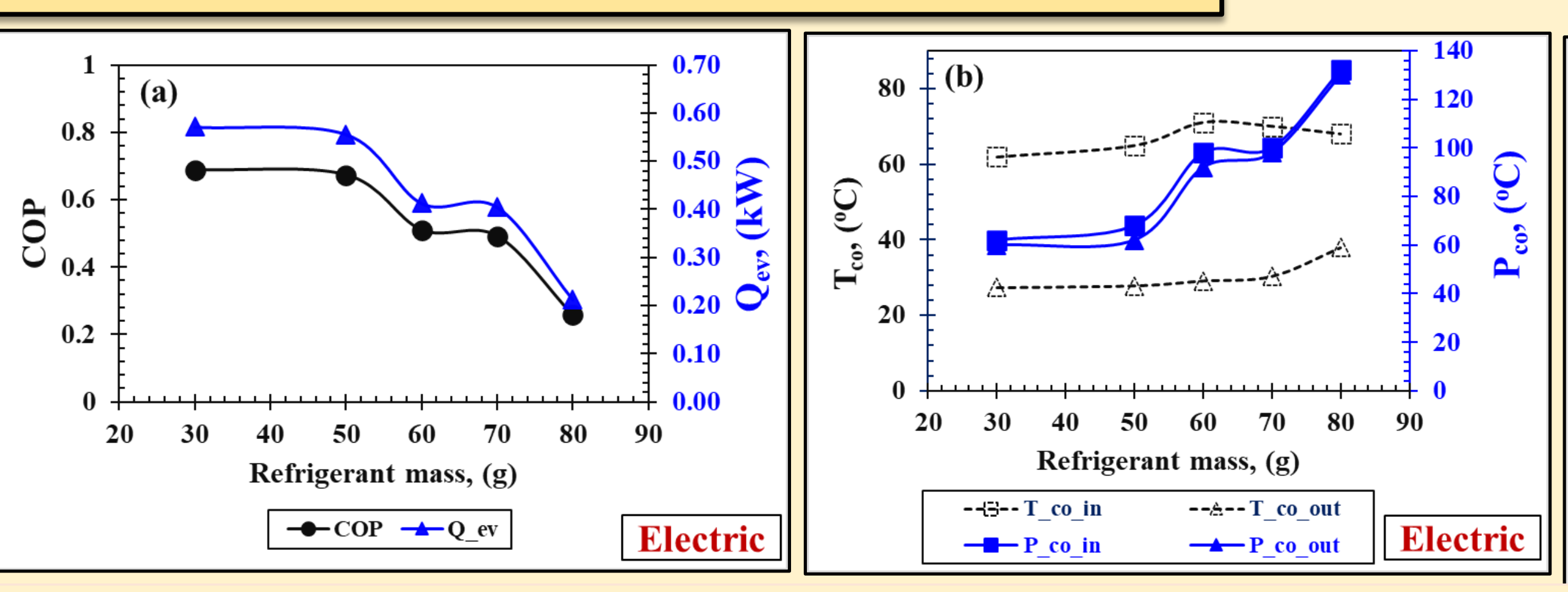

Figure 9. Experimental results of the electric operational mode.

\section{Conclusions}

An innovative TMR that works with ultra-low temperature sources (as low as $70 \mathrm{oC}$ ) using refrigerants with zero ozone depletion potential was introduced.

- A hybrid electric-pneumatic-mechanical (HEPM) refrigeration setup was built to experimentally testing and investigating the proposed TMR system and compared it to conventional electrical refrigeration system.

The experiment was performed using an ECU with diameter of $80 \mathrm{~mm}$ and stroke length of $150 \mathrm{~mm}$ for each piston., R134a as a refrigerant, supply pressure of 90psi, ECU frequency of 18rpm, and QD Series R134a Refrigeration Compressor $(220 \mathrm{~V} / 50 \mathrm{~Hz})$

The results show that the COP and $\mathrm{Q}_{\mathrm{ev}}$ of the electric-compressor mode is 1.39 and 2.61 times higher than of the ECU mode.

As a future work, to enhance the performance of the ECU compared to the electric operational, higher frequency must be applied, and the refrigerant should be changed to that works efficiently with the ECU such as (R1234yf).

\section{Acknowledgement}

This work was made possible by NPRP-S grant \# [11S-1231-170155] from the Qatar National Research Fund (a member of Qatar Foundation). The findings herein reflect the work, and are solely the responsibility, of the authors

\section{Testing procedures}

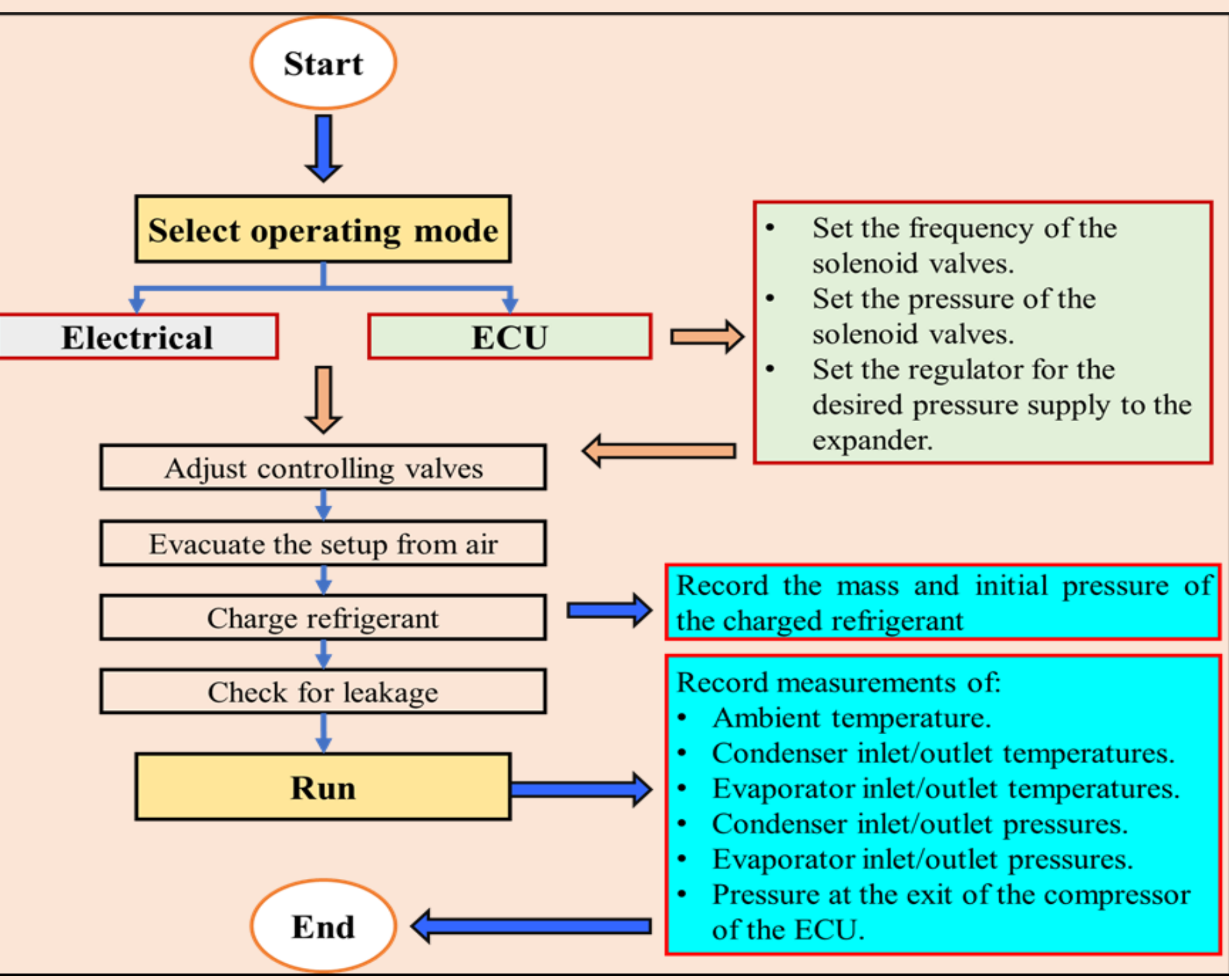

igure 6. Testing procedures of the hybrid ECU and electrical tup

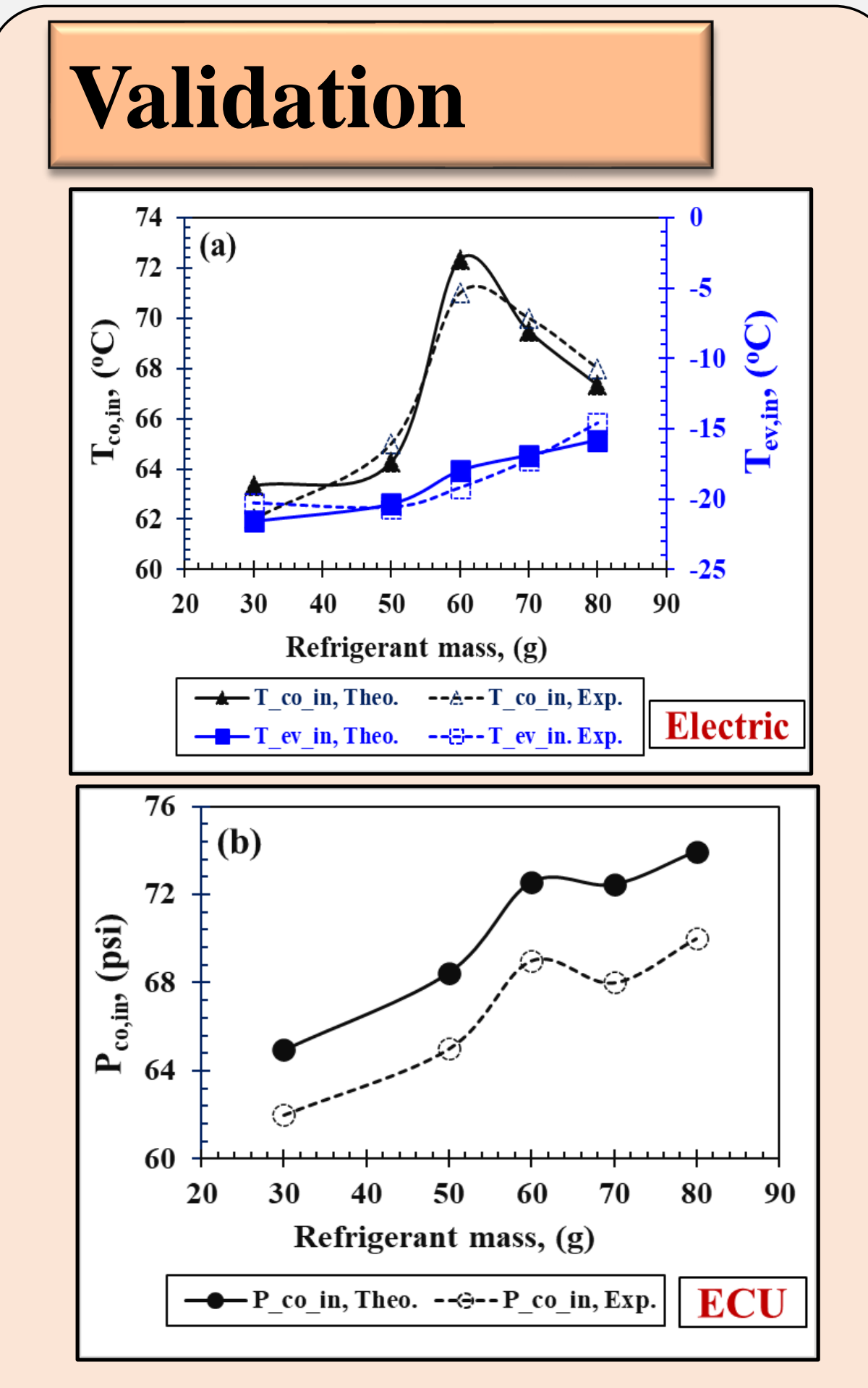

Figure 7. Validation of (a) electric

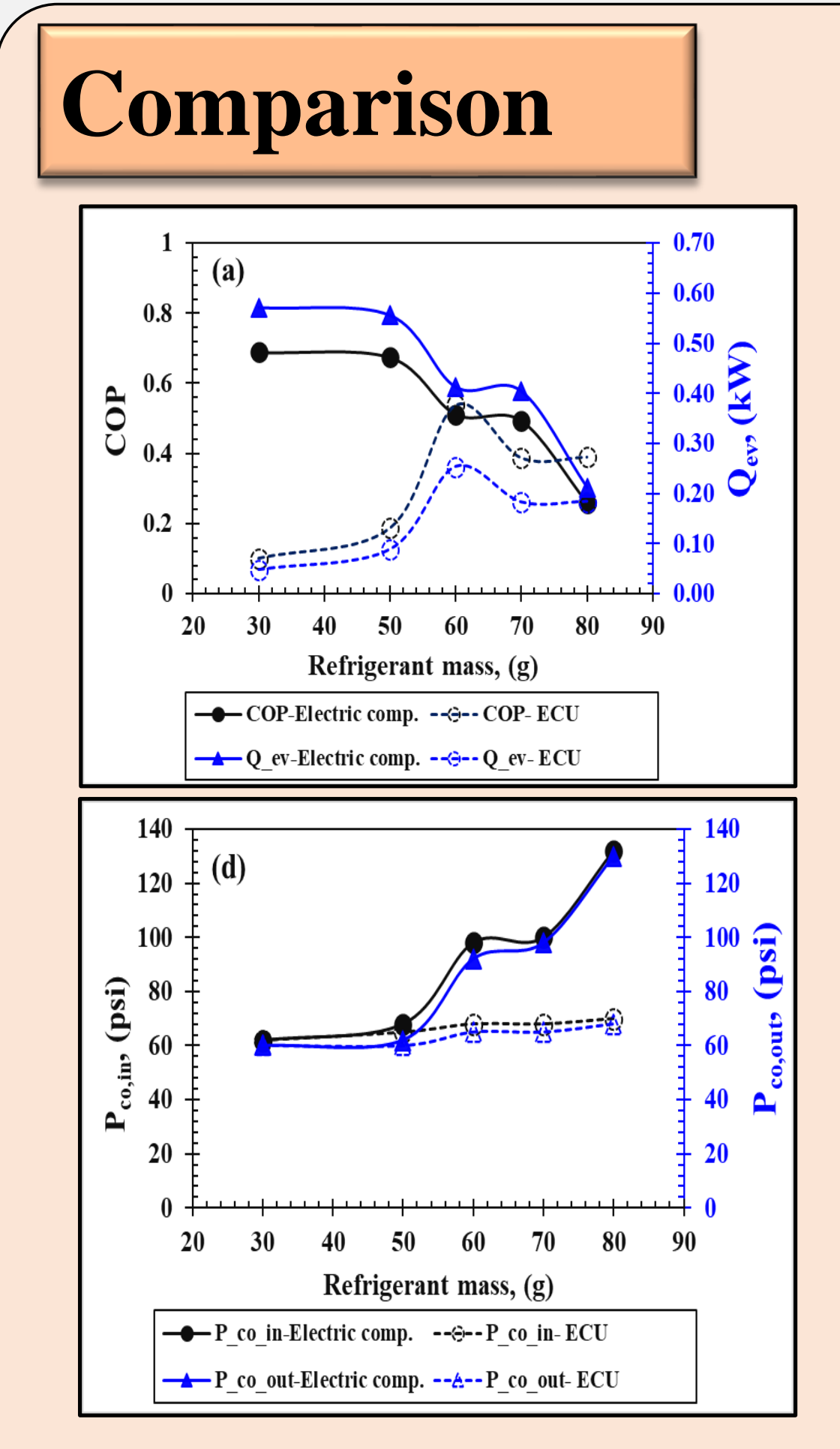

Figure 8. Comparison between the electrical and ECU operational modes.

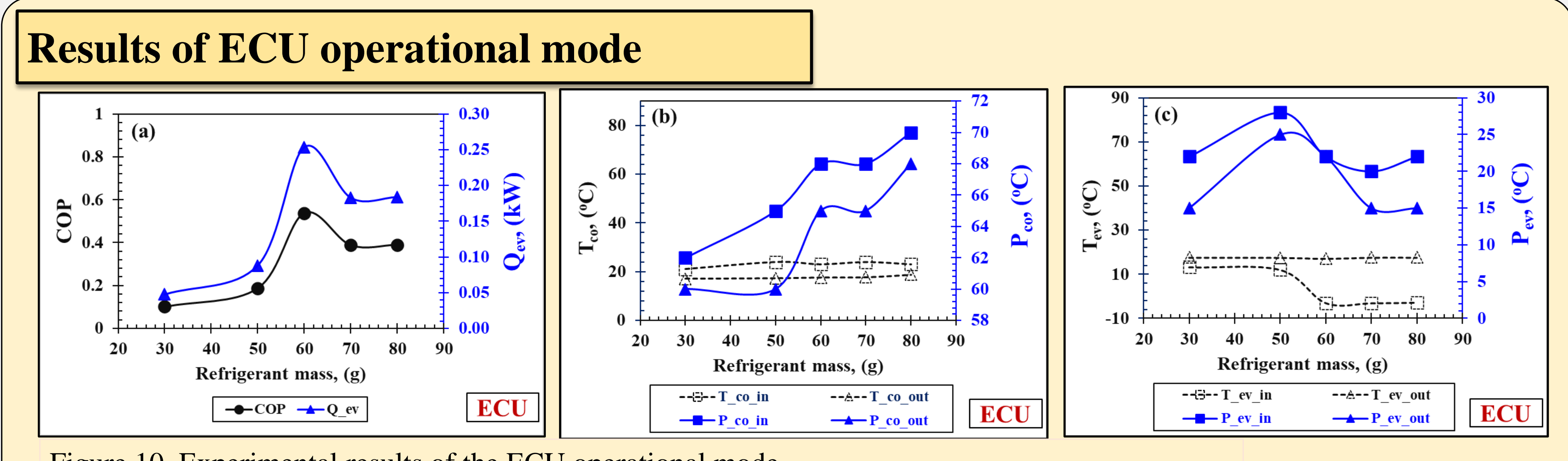

Figure 10. Experimental results of the ECU operational mode.

\section{References}
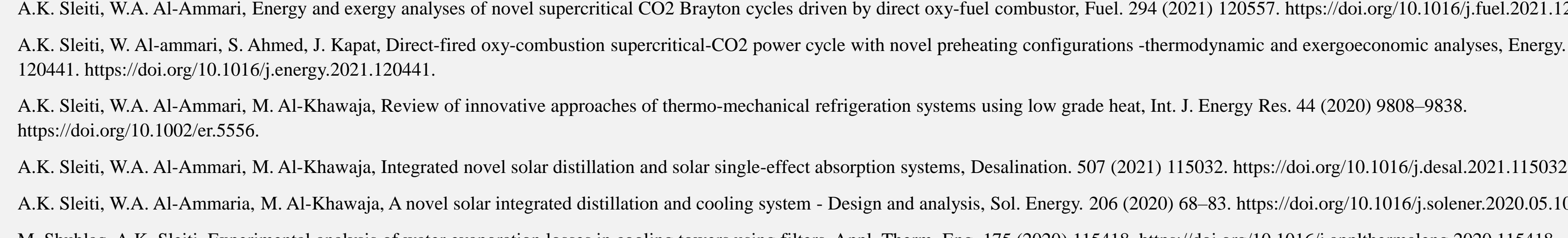

A.K. Sleiti, M. A.-Khavaja, W.A. Al-Ammari, A combin

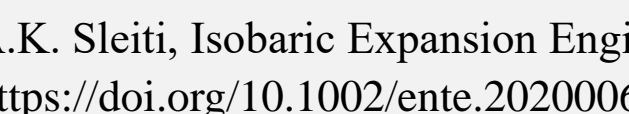

